\title{
Screening the MayBridge Rule of 3 Fragment Library for Compounds That Interact with the Trypanosoma brucei myo-Inositol-3-Phosphate Synthase and/or Show Trypanocidal Activity
}

\author{
Louise L. Major and Terry K. Smith \\ Biomolecular Science, The North Haugh, The University of St. Andrews, Fife, Scotland, KY16 9ST, UK \\ Correspondence should be addressed to Terry K. Smith, tks1@st-andrews.ac.uk
}

Received 31 December 2010; Revised 23 February 2011; Accepted 23 February 2011

Academic Editor: Wanderley De Souza

Copyright ( $) 2011$ L. L. Major and T. K. Smith. This is an open access article distributed under the Creative Commons Attribution License, which permits unrestricted use, distribution, and reproduction in any medium, provided the original work is properly cited.

\begin{abstract}
Inositol-3-phosphate synthase (INO1) has previously been genetically validated as a drug target against Trypanosoma brucei, the causative agent of African sleeping sickness. Chemical intervention of this essential enzyme could lead to new therapeutic agents. Unfortunately, no potent inhibitors of INO1 from any organism have been reported, so a screen for potential novel inhibitors of T. brucei INO1was undertaken. Detection of inhibition of T. brucei INO1 is problematic due to the nature of the reaction. Direct detection requires differentiation between glucose-6-phosphate and inositol-3-phosphate. Coupled enzyme assays could give false positives as potentially they could inhibit the coupling enzyme. Thus, an alternative approach of differential scanning fluorimetry to identify compounds that interact with T. brucei INO1 was employed to screen $\sim 670$ compounds from the MayBridge Rule of 3 Fragment Library. This approach identified 38 compounds, which significantly altered the $\mathrm{T}_{\mathrm{m}}$ of TbINO1. Four compounds showed trypanocidal activity with ED50s in the tens of micromolar range, with 2 having a selectivity index in excess of 250 . The trypanocidal and general cytotoxicity activities of all of the compounds in the library are also reported, with the best having ED50S of $\sim 20 \mu \mathrm{M}$ against $T$. brucei.
\end{abstract}

\section{Introduction}

Human African Trypanosomiasis (HAT), also called African sleeping sickness, is caused by the extracellular protozoan parasite Trypanosoma brucei. HAT is a potentially fatal disease with 200000 new cases per year in sub-Saharan Africa [1]. Despite this, current drugs are often toxic and difficult to administer, highlighting the urgent need for new, more effective drug therapies. T. brucei is able to survive in the hosts' bloodstream due to a dense coat $\left(5 \times 10^{6} \mathrm{dim}\right.$ ers/cell) of variant surface glycoprotein (VSG) [2, 3]. This coat acts as a diffusion barrier and enables the cell to avoid the hosts' innate immune system by a specialised process of antigenic variation, utilising a repertoire of more than 1000 different VSG genes $[4,5]$. Although the VSG coat is systematically changed, it is always attached to the plasma membrane via a glycosylphosphatidylinositol (GPI) anchor [6, 7].
GPI anchors are ubiquitous to eukaryotes and comprise of the basic core structure of $\mathrm{NH}_{2} \mathrm{CH}_{2} \mathrm{CH}_{2} \mathrm{PO}_{4} \mathrm{H}-6 \mathrm{Man} \alpha 1-$ 2Man $\alpha 1-6 \mathrm{Man} \alpha 1-4 \mathrm{GlcN} \alpha 1-6 \underline{\mathrm{D}}-m y o$-inositol-1- $\mathrm{HPO}_{4}$-lipid (EtN-P-Man ${ }_{3} \mathrm{GlcN}-\mathrm{PI}$ ), with a lipid moiety of diacylglycerol, alkylacylglycerol, or ceramide [8]. Previously, the biosynthesis of GPI anchors in bloodstream form T. brucei has been both genetically and chemically validated as a therapeutic drug target [9-11].

The de novo synthesis of myo-inositol is a ubiquitous process occurring in almost all eukaryotes studied. It is the result of the concerted actions of two enzymes: firstly an D-myo-inositol-3-phosphate synthase (INO1) which isomerases glucose-6-phosphate to D-myo-inositol-3-phosphate and secondly, an inositol monophosphatase which dephosphorylates the $\underline{\mathrm{D}}$-myo-inositol-3-phosphate to yield myo-inositol [12]. Previously, through the creation of a conditional knockout cell line of INO1, it was demonstrated that 
the de novo synthesis of myo-inositol is essential to the survival of bloodstream form T. brucei $[13,14]$. Surprisingly, the deletion of INO1 could not be overcome by the inclusion of extra myo-inositol in the media, which is in striking contrast to all other INO1 null mutants described to date. Intriguingly, further analysis showed that there was no significant decrease in the level of myo-inositol in the conditional knockout cells grown under nonpermissive conditions, showing that the cells were not exhibiting the typical "inositol-less" death phenotype described for all other INO1 mutants $[12,15,16]$. In vivo labelling and localisation studies of INO1 $[13,14]$ and the T. brucei phosphatidylinositol synthase [17] suggested that the de novo synthesised myoinositol is the primary source of myo-inositol used in the formation of phosphatidylinositol for GPI anchors and that there was a distinction or compartmentalisation of the de novo synthesised myo-inositol from that obtained from extracellular sources. The clear dependence by bloodstream form T. brucei on de novo synthesised myo-inositol for GPI anchor biosynthesis has not been described for any other organism to date and shows a unique avenue which could be exploited for future therapeutic drug design.

The MayBridge Rule of 3 Fragment Library (May Ro3) is a relatively small collection of chemical entries that are pharmacophore rich. The rule of 3 refers to compliance to the following criteria: $\mathrm{MW} \leq 300, \operatorname{cog} \mathrm{P} \leq 3.0, \mathrm{H}-$ Bond Acceptors $\leq 3$, H-Bond Donors $\leq 3$, Rotatable bonds (Flexibility Index) $\leq 3$, and Polar Surface Area $\leq 60 \AA$ [18]. The library has quantifiable diversity through the application of standard chemometrics, assured aqueous solubility to $\geq 1 \mathrm{mM}$ using LogS and high purity ( $\geq 95 \%)$.

As T. brucei INO1 (TbINO1) is a genetically validated drug target and screening for inhibitors is problematic due to the nature of the reaction and/or the use of a coupled enzyme assay, differential scanning fluorimetry was employed to look for compounds that interact with TbINO1. Thus, $\sim 670$ compounds from the May Ro3 fragment library were screened and their trypanocidal and general cytotoxic activities determined.

\section{Experimental}

2.1. Materials. All materials unless stated were purchased either from Sigma/Aldrich or Invitrogen. Access to the Maybridge Rule of 3 (May Ro3) library, was kindly provided by Dr Rupert Russell (St Andrews). Stock solutions of the compounds $(2 \mathrm{M})$ were prepared in DMSO and kept in master plates at $200 \mathrm{mM}$ in DMSO (100\%) by Dr Margaret Taylor (St Andrews). These were replated into daughter (working) plates occupying the central 80 wells of a 96-well plate, at $10 \mathrm{mM}$ in $5 \%$ DMSO, allowing the two outside columns for positive and negative controls.

2.2. TbINO1 Protein Overexpression and Purification. Largescale recombinant expression and purification of TbINO1 was conducted using the construct pET15b-TbINO1 in BL21 Rosetta (DE3) cells, and TbINO protein was purified by $\mathrm{Ni}$ affinity chromatography, eluted with $100 \mathrm{mM}$ imidazole,

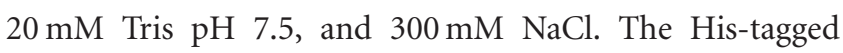

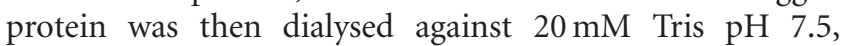
$50 \mathrm{mM} \mathrm{NaCl}, 5 \mathrm{mM}$ DTT and stored with $20 \%$ glycerol, at $-80^{\circ} \mathrm{C}$ for up to 12 months without loss of activity. Full details of expression vector construction and purification will be published elsewhere (Martin, K. L. and T. K. Smith unpublished).

\subsection{Differential Scanning Fluorimetry with TbINO1. Differ-} ential scanning fluorimetry was set up in 96-well PCR plates using a reaction volume of $100 \mu \mathrm{L}$. Shifts in TbINO1 $\mathrm{T}_{\mathrm{m}}$ with ligand binding were observed when ammonium acetate and $\mathrm{NAD}^{+}$were present. Samples contained $2 \mu \mathrm{M}$ TbINO1, $2 \mathrm{mM}$ Ammonium Acetate, $1 \mathrm{mM} \mathrm{NAD}+10 \mathrm{mM}$ HEPES $\mathrm{pH} 7.5,50 \mathrm{mM} \mathrm{NaCl}$, and 1.25 working stock of Sypro Orange (Invitrogen, as a 5000 times stock). Compounds from the May Ro3 fragment library $(1 \mathrm{mM})$ and positive controls, glucose-6-phosphate as substrate $(5 \mathrm{mM})$ and 2deoxy-glucose-6-phosphate as inhibitor $(4 \mathrm{mM})$ were added as required.

Differential fluorimetric scans were performed in a realtime PCR machine (Stratagene Mx3005P with software MxPro v 4.01) using a temperature scan from $25^{\circ} \mathrm{C}$ to $95^{\circ} \mathrm{C}$ at $0.5^{\circ} \mathrm{C} \mathrm{min}^{-1}$. Data were then exported to Excel for analysis using "DSF analysis" modified from the template provided by Niesen et al. [19]. $T_{m}$ values were calculated by nonlinear regression, fitting the Boltzmann equation to the denaturation curves using GraFit.

2.4. Cytotoxicity Studies. The trypanocidal activity of all compounds (final $0.5 \mathrm{mM}, 0.5 \% \mathrm{DMSO}$ ) against cultured bloodstream T. brucei (strain 427) was determined using the Alamar Blue viability test as described previously [20].

Cytotoxic effects against HeLa and A549 cells were determined in a similar manner. Briefly, the cells were cultured in DMEM supplemented with $10 \%$ FCS and $2 \mathrm{mM} \mathrm{L-}$ Glutamine. Cells were plated at initial cell concentration of $2 \times 10^{4}$ cells/well and incubated with the compounds for $\sim 65$ hours prior to addition of Alamar Blue solution for a further 5 hours.

\section{Results and Discussion}

3.1. Is TbINO1 Amenable to Differential Scanning Fluorimetry? . Inositol-3-phosphate synthase has previously been genetically validated as a drug target against $T$. brucei $[13,14]$, and is a prime candidate for chemical intervention as a therapeutic against African sleeping sickness. Unfortunately, no potent inhibitors of INO1 from any organism have been reported; therefore, it was decided to undertake a screen for potential novel inhibitors of TbINO1. Screening for inhibitors of TbINO1 is problematic due to the difficulty in following the reaction, that is, having to directly differentiate between glucose-6-phosphate and inositol-3-phosphate, or alternatively using a coupled enzyme assay, where a compound could potentially inhibit the coupled enzyme [14]. Thus, an alternative approach was taken, using differential scanning fluorimetry. Differential scanning fluorimetry has 


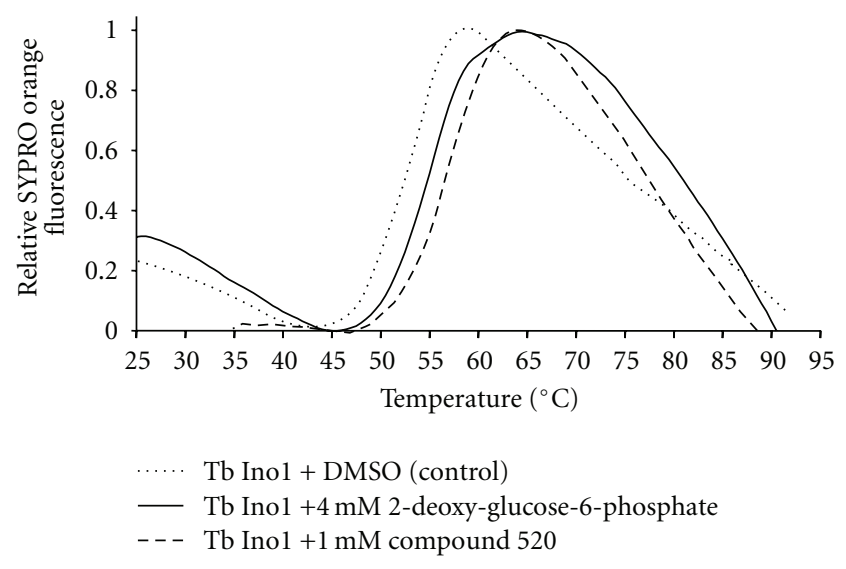

FIgURE 1: Typical differential fluorimetry scans of TbINO1. Differential fluorimetric scans were performed and analysed as described in Experimental. TbINO1 + DMSO (control) dotted line, TbINO1 + 4 mM 2-deoxy-glucose-6-phosphate (positive control) solid line, and TbINO1 $+1 \mathrm{mM}$ compound 520, dashed line.

been used to identify compounds that interact with a protein, either to stabilise or destabilise it, and therefore influencing the protein's $\mathrm{T}_{\mathrm{m}}$ (melting point) [21].

TbINO1 was subjected to differential scanning fluorimetry to ascertain if this approach was possible. The $T_{m}$ for TbINO1 was determined in the presence of $\mathrm{NAD}^{+}$and ammonium acetate, both known cofactors and stimuli of INO1 activity $[13,14,22]$; in a typical experiment, $\mathrm{T}_{\mathrm{m}} \mathrm{s}$ were consistently $\sim 51.4^{\circ} \mathrm{C}\left(4\right.$ samples, range $\left.51.36-51.39^{\circ} \mathrm{C}\right)$ (Figure 1, dotted line). The substrate glucose-6-phosphate $(5 \mathrm{mM})$ increased the $\mathrm{T}_{\mathrm{m}}$ by $0.70 \pm 0.28^{\circ} \mathrm{C}$; however, 2deoxy-glucose-6-phosphate $(4 \mathrm{mM})$, a known inhibitor of INO1s [22-24], increased the $\mathrm{T}_{\mathrm{m}}$ value by $+2.84 \pm 0.47^{\circ} \mathrm{C}$ (Figure 1, solid line). These encouraging results allowed validation of a screening program; thus, glucose-6-phosphate and 2-deoxy-glucose-6-phosphate were used as positive controls, and DMSO (in which all compounds were dissolved) as a negative control on all subsequent screening plates.

3.2. Screening of the May Ro3 Fragment Library for Compounds That Interact with Tbino1 by Differential Scanning Fluorimetry. As TbINO1 was amenable to screening by differential scanning fluorimetry, $\sim 670$ compounds from the May Ro3 fragment library were screened. Additionally, their trypanocidal and general cytotoxicity activities, against bloodstream T. brucei and HeLa and A549 cells were assessed (see Supplementary Table 1 in Supplementary Material available online at doi: 10.4061/2011/389364).

From this large amount of data, 38 compounds at $1 \mathrm{mM}$ interacted with TbINO1 with a $\Delta \mathrm{T}_{\mathrm{m}}>+1.5^{\circ} \mathrm{C}$ (Table 1). Of these compound 520, 2-(2-furyl)benzoic acid, stabilised the protein the greatest with a $\Delta \mathrm{T}_{\mathrm{m}}+4.29 \pm 0.07^{\circ} \mathrm{C}$ (Figure 1 , dashed line). It is interesting to note that the heterocyclic furan moiety is a common feature in several of the top hits (Table 1, compounds 520, 75, 30, 28, and 383).

Other similarities between the top hits are the presence of a carboxylic acid (Table 1, compounds 520, 75, 28, 513) or a methanol group (Table 1, compounds 186, 30, 383) attached to an aromatic ring. An obvious conclusion is that these moieties form important hydrogen bonds to the protein, in a similar orientation to each other with respect to the aromatic ring to which they are attached.

The trypanocidal and cytotoxicity activities of these 38 compounds (Table 1) revealed that 9 of them killed more than $35 \%$ of $T$. brucei at $0.5 \mathrm{mM}$. These were investigated further, and their ED50s for both T. brucei and HeLa cells were determined (Table 1). Of these compound 162, 1Hindol-3-ylmethanol with a $\Delta \mathrm{T}_{\mathrm{m}}+2.31 \pm 0.06^{\circ} \mathrm{C}$ (Table 1), was the most potent with an ED50 of $31 \pm 1.4 \mu \mathrm{M}$, but was also cytotoxic against HeLa cells, ED50 of $103 \pm 6 \mu \mathrm{M}$, thus giving a very poor selectivity index of 3.3. However, another compound 256, 2-quinolinylmethanol, structurally very similar to compound 162, that has an ED50 of $40.1 \pm 1.2 \mu \mathrm{M}$, showed less cytotoxicity against HeLa cells, giving a selectivity index of $\sim 21$.

Interestingly, the top TbINO1 differential scanning fluorimetry hit, compound 520, also showed trypanocidal activity, ED50 of $76 \pm 6 \mu \mathrm{M}$, but no cytotoxicity towards HeLa cells at $20 \mathrm{mM}$, thus giving a selectivity index $>256$. However, the best selectivity index from these TbINO1 differential scanning fluorimetry hits (Table 1) was compound 239, 2amino-4-methylthiophene-3-carboxamide, with a T. brucei ED50 of $63 \pm 2.5 \mu \mathrm{M}$ and a selectivity index $>317$.

It is interesting to note that compound 520 contains a carboxylic acid and, therefore, should be impervious to membranes by passive diffusion; this seems to be true for the close analogous compound 513, 2-(1H-pyrrol-1-yl)benzoic acid, which also interacts with TbINO1 strongly but has no trypanocidal or general cytotoxicity activity at $0.5 \mathrm{mM}$ (Table 1).

Puzzlingly, this suggests that some carboxylic acid containing compounds, such as 520 , may be specifically and actively taken up by T. brucei, while other closely related carboxylic acid containing compounds are not.

The TbINO1 differential scanning fluorimetry hits will be investigated further in the future, as outlined in the conclusions.

3.3. Screening of the May Ro3 Fragment Library for Trypanocidal Compounds. The most potent trypanocidal compounds (ED50s $<100 \mu \mathrm{M})$ of the $\sim 670$ compounds from the May Ro3 fragment library (Supplementary Table 1) were investigated further by determining their ED50s in both T. brucei and HeLa cells (Table 2). From first observations, it is clear that the vast majority of these compounds contain a primary amine, with the most potent compounds (ED50s $<40 \mu \mathrm{M}$ ), 269,270 , and 348 , containing an aromatic primary amine. Unfortunately, most of the primary amine containing compounds are cytotoxic and thus have poor selectivity indexes (0.5-21). The only exceptions are compounds 520 and 239 (both of which do not contain a primary amine), as discussed earlier, and showed no cytotoxicity at $20 \mathrm{mM}$ (Tables 1 and 2) with selectivity indexes greater than 260 .

The structure activity relationship of the most potent trypanocidal compound from the library, compound 269 


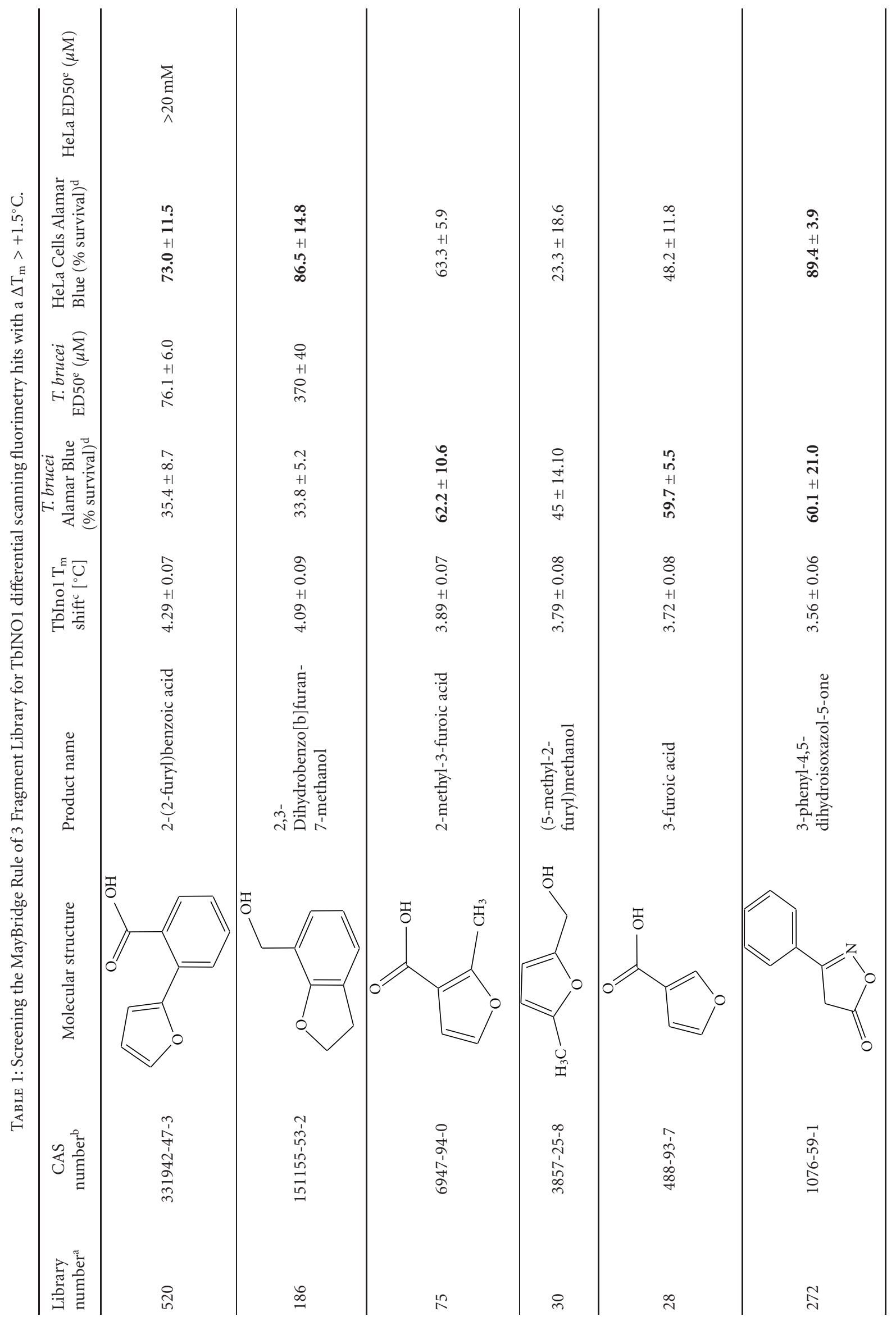




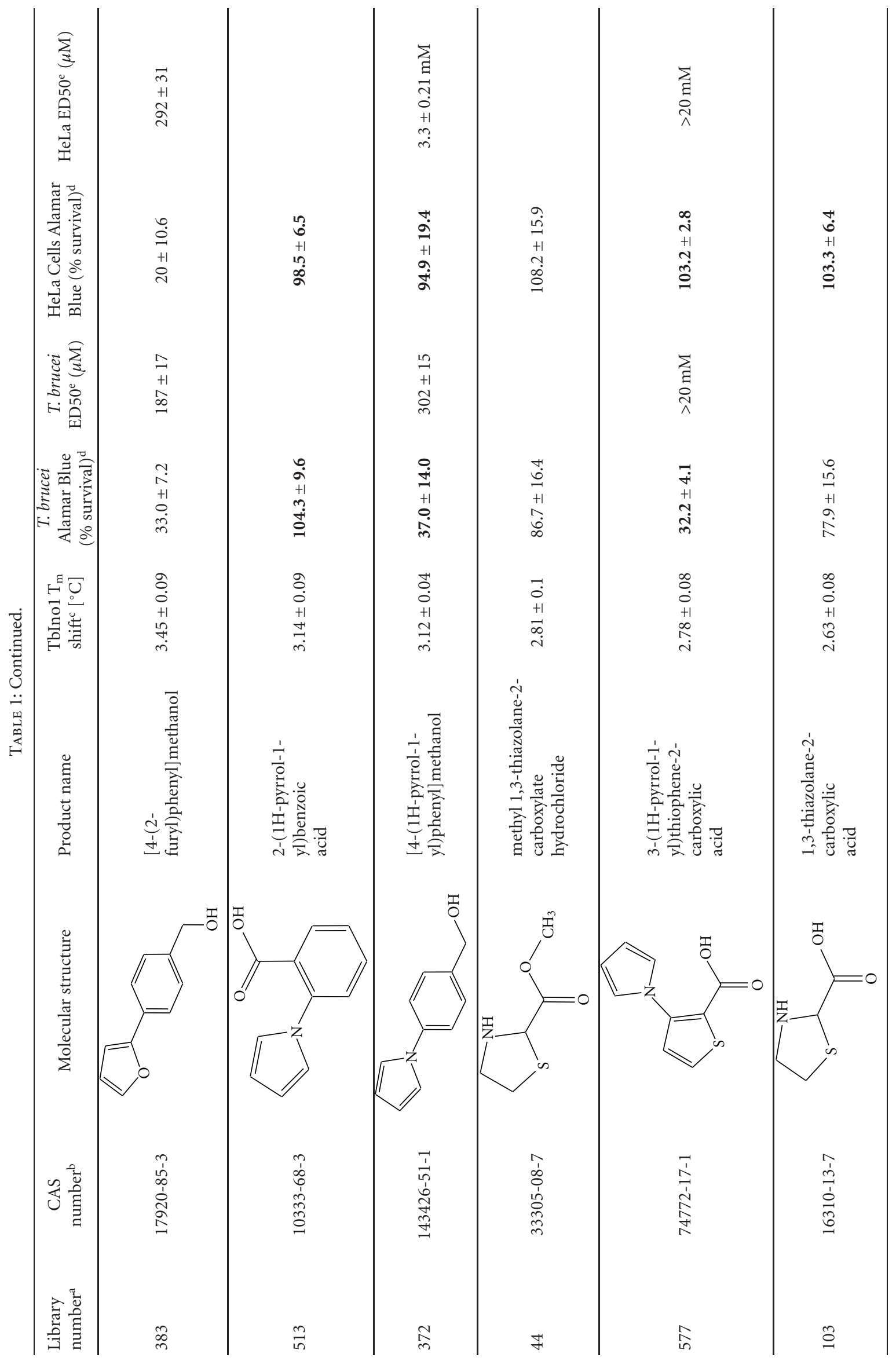




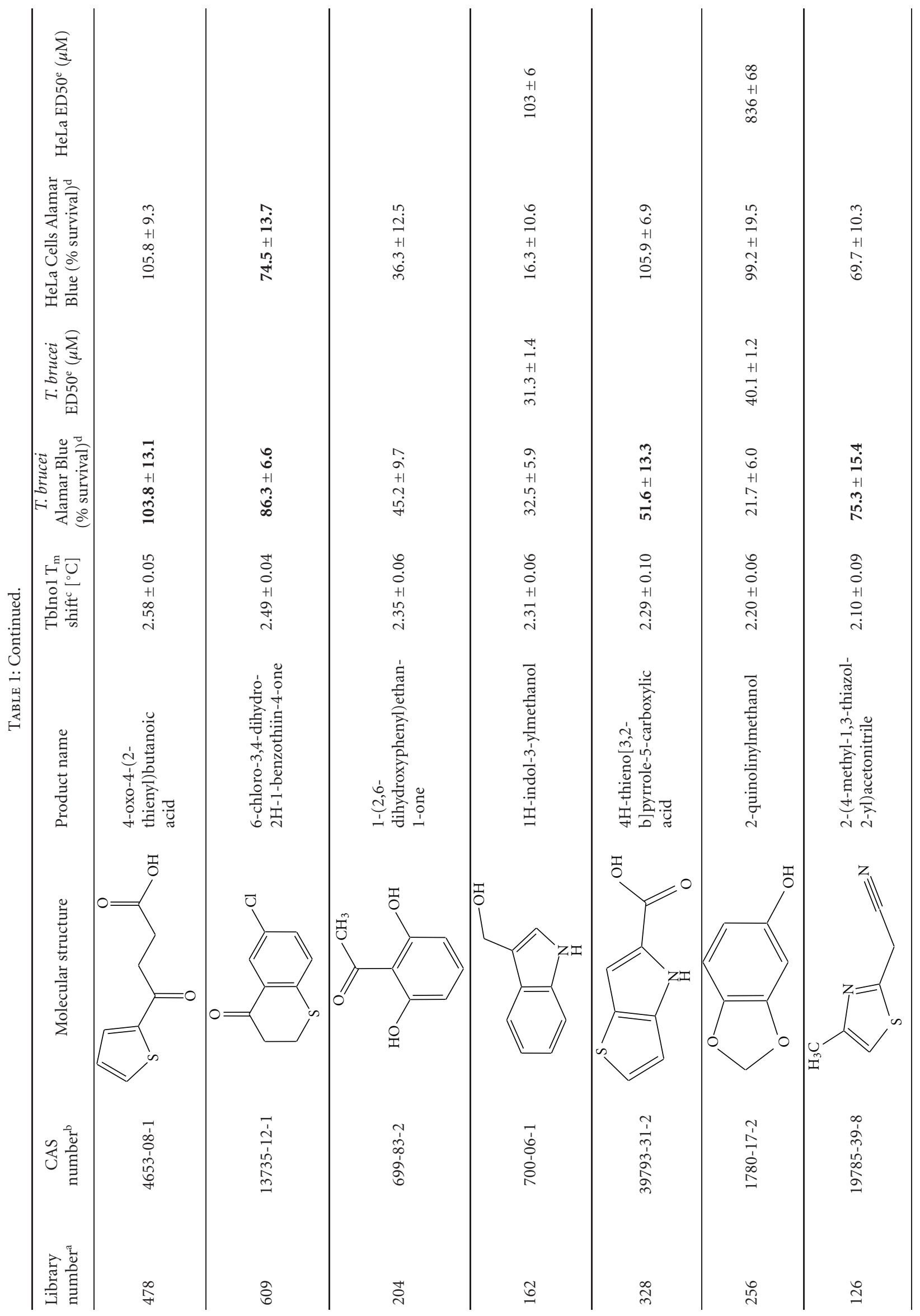




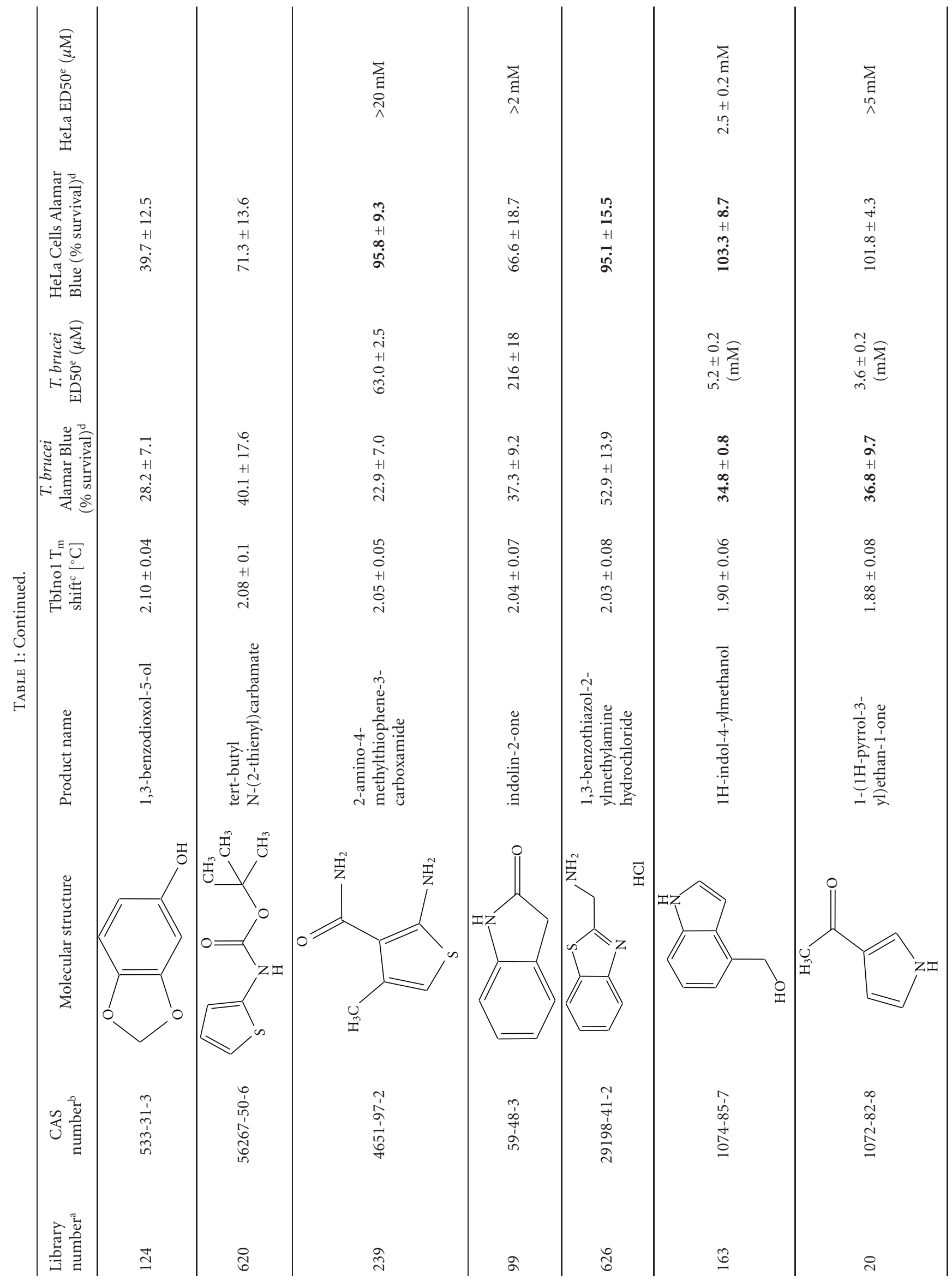




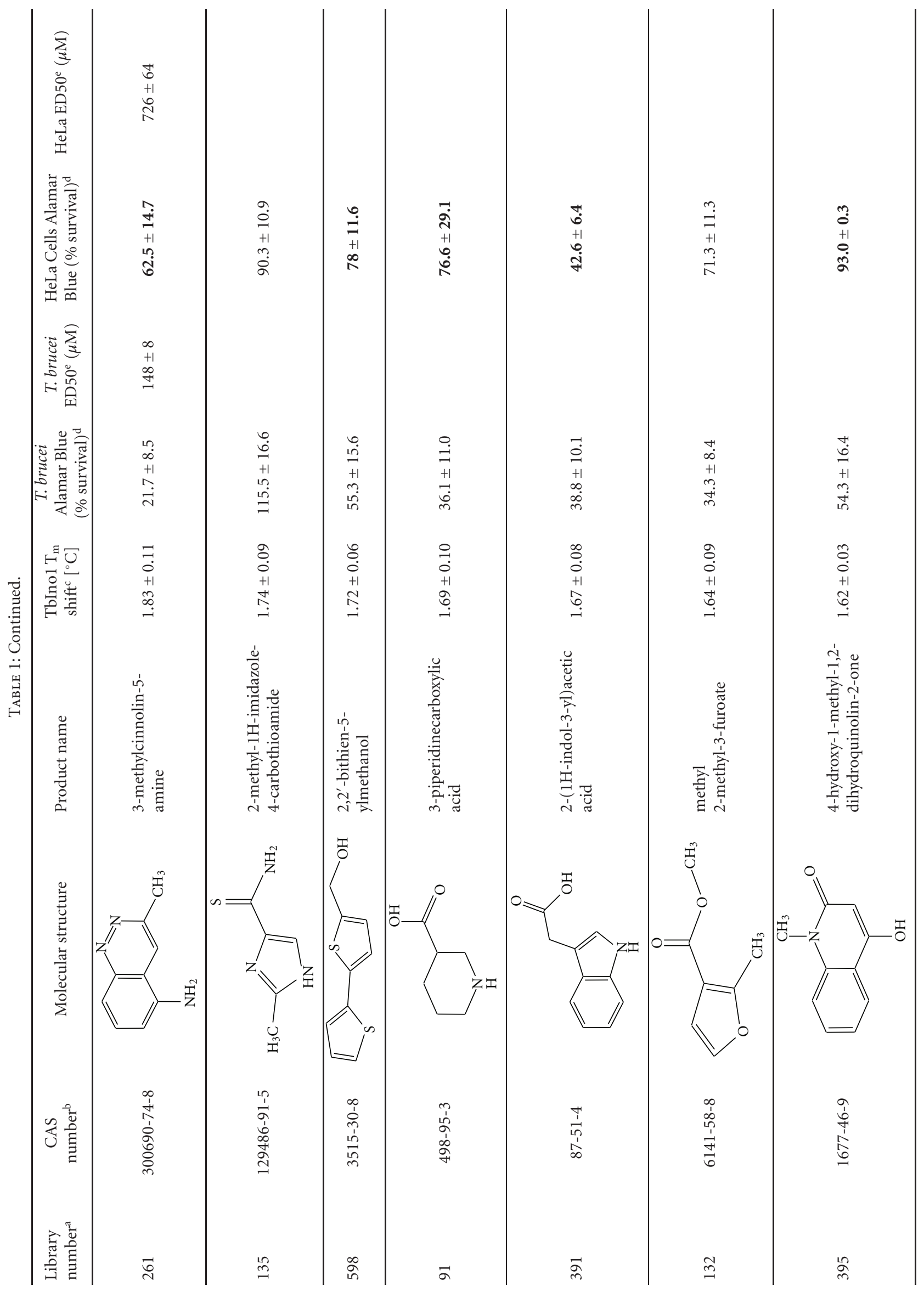




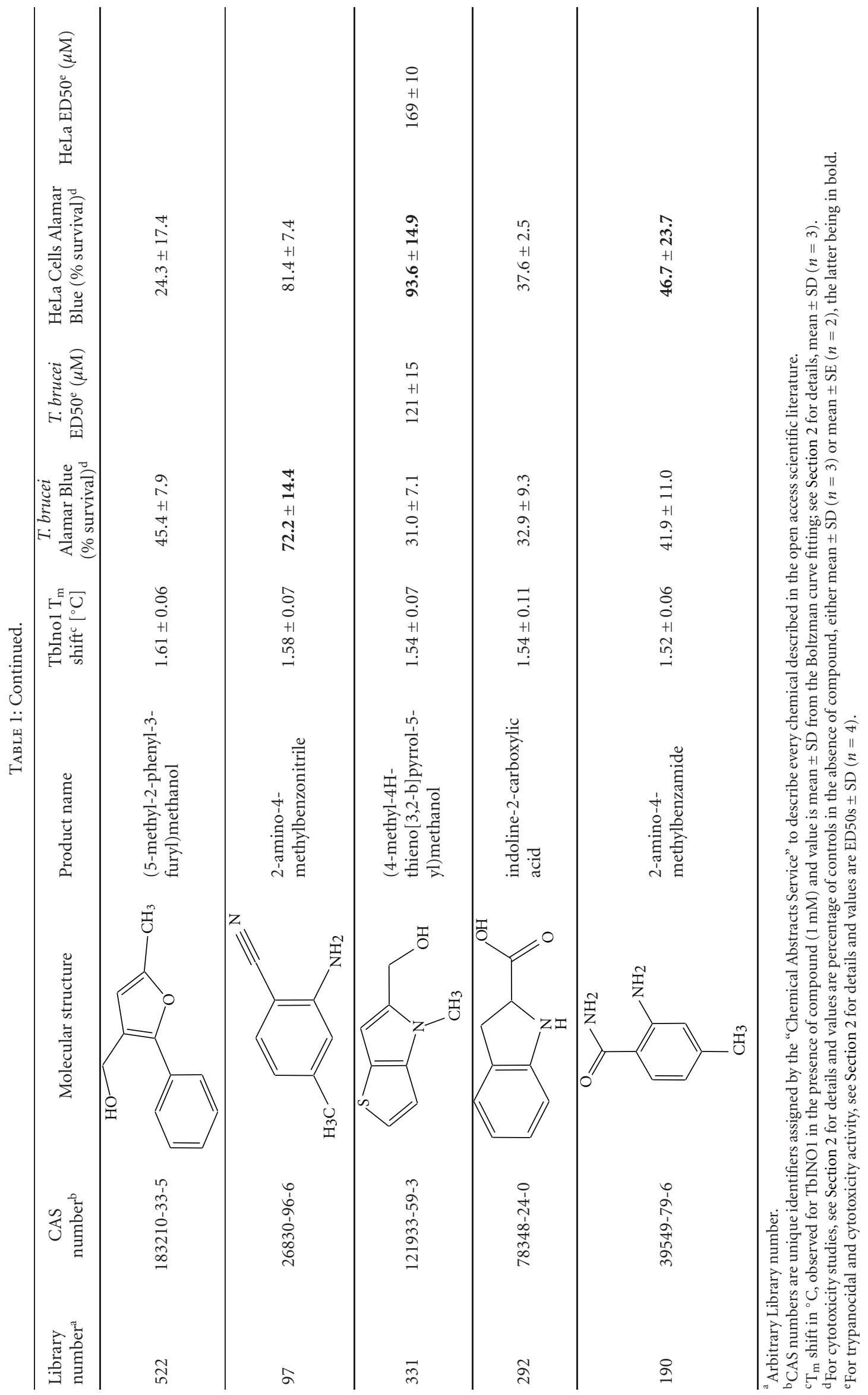


TABLE 2: Screening for trypanocidal compounds in the MayBridge Rule of 3 Fragment Library.

\begin{tabular}{|c|c|c|c|c|}
\hline Library number $^{\mathrm{a}}$ & Molecular structure & T. brucei ED50 ${ }^{\mathrm{b}} \quad(\mu \mathrm{M})$ & HeLa ED50 $(\mu \mathrm{M})$ & Selectivity index \\
\hline 269 & & $20.1 \pm 1.0$ & $431 \pm 53$ & 21.4 \\
\hline 270 & & $30.5 \pm 1.6$ & $206 \pm 13$ & 6.8 \\
\hline 162 & & $31 \pm 1.4$ & $103 \pm 6$ & 3.3 \\
\hline 348 & & $35.0 \pm 1.0$ & $21.8 \pm 1.8$ & 0.6 \\
\hline 256 & & $40.1 \pm 1.2$ & $836 \pm 68$ & 20.8 \\
\hline 281 & & $44.7 \pm 1.8$ & $659 \pm 65$ & 14.7 \\
\hline 365 & & $49.4 \pm 2.1$ & $71.8 \pm 3.7$ & 1.5 \\
\hline 213 & & $55.6 \pm 1.3$ & $710 \pm 96$ & 12.8 \\
\hline 257 & & $56.0 \pm 3.6$ & $114 \pm 8$ & 2.0 \\
\hline 239 & & $63.0 \pm 2.5$ & $>20 \mathrm{mM}$ & $>317$ \\
\hline
\end{tabular}


TABle 2: Continued.

\begin{tabular}{llll}
\hline Library number $^{\mathrm{a}}$ & T. brucei $\mathrm{ED} 50^{\mathrm{b}}(\mu \mathrm{M})$ & HeLa ED50 $(\mu \mathrm{M})$ & Selectivity index \\
\hline 250 & & & \\
\hline
\end{tabular}

${ }^{a}$ Arbitrary Library number.

${ }^{\mathrm{b}}$ For trypanocidal and cytotoxicity activity, see Section 2 for details and values are ED50s $\pm \operatorname{SD}(n=4)$.

'Selectivity index: T. brucei ED50/HeLa ED50.

(4-chloro-5-fluorobenzene-1,2-diamine), with an ED50 of $20.1 \pm 1.03 \mu \mathrm{M}$, was investigated further. A series of analogues were purchased and tested in parallel; this included analogues that had the two amino groups, but with only one of the halides or one of the halides replaced by a methyl group or no halides. Other analogues maintained the halides, and reduce the amino groups to one, or by masking both free amines as a benzimidazole.

T. brucei and HeLa cell ED50s values were determined for this collection of compounds (Table 3 ).

Repurchased compound 269, gave ED50s similar to those obtained earlier $(20.1 \pm 1.03$ and $31.1 \pm 1.22 \mu \mathrm{M})$. As to the structure activity relationship of the analogues, it is apparent that the two amino groups seem to be important for potent trypanocidal activity, as both compounds $269 \mathrm{~b}$ and $269 \mathrm{~d}$ have dramatically increased ED50s.

The presence of two bulky groups opposite to the amine groups seems detrimental as shown by compound 240 (4chloro-5-methylbenzene-1,2-diamine); a compound from the May Ro3 fragment library (Supplementary Table 1).

The absence of one or both of the halides does not alter the T. brucei ED50s dramatically, as demonstrated by compound 269a (benzene-1,2-diamine), with an ED50 of $35.2 \pm 3.0 \mu \mathrm{M}$ (Table 3 ). The HeLa cell cytotoxicity of various benzene-1,2-diamine analogues varies dramatically, however the selectivity indexes are poor, the best being the original compound 269, 4-chloro-5-fluorobenzene-1,2diamine, with a selectivity index of $\sim 21$ (Table 3 )

Unfortunately, the benzene-1,2-diamine analogues are thought to be carcinogenic, as they are suspected to be able to interchelate DNA, although direct proof of this is still lacking [25]. These diamines are also well known to form Schiff bases and are often used for derivatisation of natural ketones and aldehydes, such as methglyoxal [26]. Ironically, it is also this Schiff base capability, which has led them to be investigated as possible anticancer, antibacterial, antifungal and antiviral agents $[27,28]$. Either or both of the DNA interchelating, or Schiff base capabilities could be the possible mode of action for the trypanocidal activity. To avoid the obvious possible problems associated with carcinogenic compounds, while still maintaining the dual functionality that seems to be important for potency, compound 269e (2-amino-1-hydroxy-benzene or 2-aminophenol), was purchased and tested. This proved to be the most potent trypanocidal agent tested in this study with an ED50 of $20.0 \pm 0.3 \mu \mathrm{M}$; however, it was cytotoxic to the HeLa cells (Table 3 ). Despite this, the relatively simple molecule (2-amino-phenol) has been shown to have anti-microbial activity $[29,30]$.

Considering these are very simple molecules, it is surprising that they are trypanocidal at low-micromolar concentrations, highlighting the importance of screening programs to identify novel pharmacophores.

\section{Conclusions}

In this work, screening of a comparatively small fragment library for thermal stabilisation of TbINO1 has allowed identification of several novel compounds that interact strongly 
TABLE 3: Structure activity relationship for trypanocidal activity of analogues of the diamine compound 269.

\begin{tabular}{|c|c|c|c|c|c|}
\hline Library number ${ }^{\mathrm{a}}$ & CAS number ${ }^{\mathrm{b}}$ & Molecular structure & T. brucei $\operatorname{ED}^{2} 0^{\mathrm{c}}(\mu \mathrm{M})$ & $\mathrm{HeLa} \mathrm{ED}^{2} 0^{c}(\mu \mathrm{M})$ & Selectivity index ${ }^{\mathrm{d}}$ \\
\hline 269 & $139512-70-2$ & & $20.1 \pm 1.2$ & $431 \pm 53$ & 21.4 \\
\hline $269 \mathrm{e}$ & $51-19-4$ or $95-55-6$ & & $20.0 \pm 0.3$ & $68.2 \pm 3.2$ & 3.4 \\
\hline $269 c$ & $95-83-0$ & & $21.9 \pm 1.2$ & $224 \pm 35$ & 10.2 \\
\hline $269 a$ & $95-54-5$ & & $35.2 \pm 3.0$ & $32.9 \pm 2.5$ & 0.9 \\
\hline 240 & $63155-04-4$ & & $86.6 \pm 4.8$ & $454 \pm 55$ & 5.2 \\
\hline $269 b$ & $367-22-6$ & & $150 \pm 13$ & $1.4 \pm 0.1 \mathrm{mM}$ & 9.3 \\
\hline $269 d$ & $175135-04-3$ & & $331 \pm 24$ & $520 \pm 52$ & 1.6 \\
\hline
\end{tabular}

${ }^{a}$ Arbitrary Library number.

${ }^{b}$ CAS numbers are unique identifiers assigned by the "Chemical Abstracts Service" to describe every chemical described in the open access scientific literature.

${ }^{c}$ For trypanocidal and cytotoxicity activity, see Section 2 for details and values are ED50s $\pm \operatorname{SD}(n=4)$.

${ }^{\mathrm{d}}$ Selectivity index: T. brucei ED50/HeLa ED50.

and stabilise TbINO1. Unexpectedly, several of the significant hits are also trypanocidal with ED50 values in the 30$80 \mu \mathrm{M}$ range, despite being relative simple molecules.

Several other compounds from the May Ro3 library showed low-micromolar trypanocidal activity. The majority of the most potent hits contain primary amines whose mode of action could be via Schiff-base formation, while some of the diamines could also be acting by inter-chelating DNA, thus interfering with cell-cycle progression/cell division.

Unfortunately, some of these compounds are cytotoxic against mammalian cells and thus are unlikely to progress as lead compounds. However, the biological activity of related compounds such as 269e (2-amino-phenol), also known to have anti-microbial activity, will be investigated further.
Future work outside the scope of this study will include the following:

(i) investigating if the lead compounds that interacted with TbINO1 inhibit its activity, in an in vitro coupled enzyme assay,

(ii) investigating the mode of killing by the lead compounds that interacted with TbINO1 by undertaking various in vivo labelling experiments to ascertain if they are inhibiting TbINO1, thus causing a lack of de novo synthesised myo-inositol, required for PI synthesis for the essential GPI pathway,

(iii) the direct interactions of compounds with TbINO1 will be investigated by protein crystallography studies. 


\section{Acknowledgments}

This work was supported in part by a Wellcome Trust Senior Research Fellowship (067441) and Wellcome Trust project Grants nos. 086658 and 093228. The authors thank Dr Rupert Russell (St. Andrews) supported by SUSLA, for access to the MayBridge Rule of 3 Fragment Library.

\section{References}

[1] WHO, http://www.who.int/tdrold/diseases/tryp/diseaseinfo .htm.

[2] M. A. J. Ferguson and G. A. M. Cross, "Myristylation of the membrane form of a Trypanosoma brucei variant surface glycoprotein," Journal of Biological Chemistry, vol. 259, no. 5, pp. 3011-3015, 1984.

[3] M. A. J. Ferguson, M. G. Low, and G. A. M. Cross, "Glycosylsn-1,2-dimyristylphosphatidylinositol is covalently linked to Trypanosoma brucei variant surface glycoprotein," Journal of Biological Chemistry, vol. 260, no. 27, pp. 14547-14555, 1985.

[4] G. A. M. Cross, "Antigenic variation in trypanosomes: secrets surface slowly," BioEssays, vol. 18, no. 4, pp. 283-291, 1996.

[5] N. Aitcheson, S. Talbot, J. Shapiro et al., "VSG switching in Trypanosoma brucei: antigenic variation analysed using RNAi in the absence of immune selection," Molecular Microbiology, vol. 57, no. 6, pp. 1608-1622, 2005.

[6] M. A. J. Ferguson, M. Duszenko, and G. S. Lamont, "Biosynthesis of Trypanosoma brucei variant surface glycoproteins. NGlycosylation and addition of a phosphatidylinositol membrane anchor," Journal of Biological Chemistry, vol. 261, no. 1, pp. 356-362, 1986.

[7] M. A. J. Ferguson, J. S. Brimacombe, J. R. Brown et al., "The GPI biosynthetic pathway as a therapeutic target for African sleeping sickness," Biochimica et Biophysica Acta, vol. 1455, no. 2-3, pp. 327-340, 1999.

[8] M. A. J. Ferguson, J. S. Brimacombe, J. R. Brown et al., "The GPI biosynthetic pathway as a therapeutic target for African sleeping sickness," Biochimica et Biophysica Acta, vol. 1455, no. 2-3, pp. 327-340, 1999.

[9] T. Chang, K. G. Milne, M. L. S. Güther, T. K. Smith, and M. A. J. Ferguson, "Cloning of Trypanosoma brucei and Leishmania major genes encoding the GlcNAc-phosphatidylinositol De$\mathrm{N}$-acetylase of glycosylphosphatidylinositol biosynthesis that is essential to the African sleeping sickness parasite," Journal of Biological Chemistry, vol. 277, no. 51, pp. 50176-50182, 2002.

[10] K. Nagamune, T. Nozaki, Y. Maeda et al., "Critical roles of glycosylphosphatidylinositol for Trypanosoma brucei," Proceedings of the National Academy of Sciences of the United States of America, vol. 97, no. 19, pp. 10336-10341, 2000.

[11] T. K. Smith, A. Crossman, J. S. Brimacombe, and M. A. J. Ferguson, "Chemical validation of GPI biosynthesis as a drug target against African sleeping sickness," EMBO Journal, vol. 23, no. 23, pp. 4701-4708, 2004.

[12] S. A. Henry, K. D. Atkinson, A. I. Kolat, and M. R. Culbertson, "Growth and metabolism of inositol starved Saccharomyces cerevisiae," Journal of Bacteriology, vol. 68, pp. 2888-2898, 1977.

[13] K. L. Martin and T. K. Smith, "The myo-inositol-1-phosphate synthase gene is essential in Trypanosoma brucei," Biochemical Society Transactions, vol. 33, no. 5, pp. 983-985, 2005.

[14] K. L. Martin and T. K. Smith, "The glycosylphosphatidylinositol (GPI) biosynthetic pathway of bloodstream-form
Trypanosoma brucei is dependent on the de novo synthesis of inositol," Molecular Microbiology, vol. 61, no. 1, pp. 89-105, 2006.

[15] T. Ilg, "Generation of myo-inositol-auxotrophic Leishmania mexicana mutants by targeted replacement of the myoinositol-1-phosphate synthase gene," Molecular and Biochemical Parasitology, vol. 120, no. 1, pp. 151-156, 2002.

[16] F. Movahedzadeh, D. A. Smith, R. A. Norman et al., "The Mycobactenum tuberculosis inol gene is essential for growth and virulence," Molecular Microbiology, vol. 51, no. 4, pp. 1003-1014, 2004.

[17] K. L. Martin and T. K. Smith, "Phosphatidylinositol synthesis is essential in bloodstream form Trypanosoma brucei," Biochemical Journal, vol. 396, no. 2, pp. 287-295, 2006.

[18] R. A. E. Carr, M. Congreve, C. W. Murray, and D. C. Rees, "Fragment-based lead discovery: leads by design," Drug Discovery Today, vol. 10, no. 14, pp. 987-992, 2005.

[19] F. H. Niesen, H. Berglund, and M. Vedadi, "The use of differential scanning fluorimetry to detect ligand interactions that promote protein stability," Nature Protocols, vol. 2, no. 9, pp. 2212-2221, 2007.

[20] J. Mikus and D. Steverding, "A simple colorimetric method to screen drug cytotoxicity against Leishmania using the dye Alamar Blue," Parasitology International, vol. 48, no. 3, pp. 265-269, 2000.

[21] M. C. Lo, A. Aulabaugh, G. Jin et al., "Evaluation of fluorescence-based thermal shift assays for hit identification in drug discovery," Analytical Biochemistry, vol. 332, no. 1, pp. 153159, 2004.

[22] X. Jin, K. M. Foley, and J. H. Geiger, "The Structure of the 1L-myo-inositol-1-phosphate synthase-NAD -2-deoxy-Dglucitol 6-(E)-vinylhomophosphonate complex demands a revision of the enzyme mechanism," Journal of Biological Chemistry, vol. 279, no. 14, pp. 13889-13895, 2004.

[23] K. Neelon, Y. Wang, B. Stec, and M. F. Roberts, "Probing the mechanism of the Archaeoglobus fulgidus inositol-1phosphate synthase," Journal of Biological Chemistry, vol. 280, no. 12, pp. 11475-11482, 2005.

[24] K. A. Stieglitz, H. Yang, M. F. Roberts, and B. Stec, "Reaching for mechanistic consensus across life kingdoms: structure and insights into catalysis of the myo-Inositol-1-phosphate synthase (mIPS) from Archaeoglobus fulgidus," Biochemistry, vol. 44, no. 1, pp. 213-224, 2005.

[25] E. K. Weisburger, A. S. K. Murthy, R. W. Fleischman, and M. Hagopian, "Carcinogenicity of 4-chloro-o-phenylenediamine, 4-chloro- $m$-phenylenediamine, and 2-chloro-p-phenylenediamine in Fischer 344 rats and B6C3F mice," Carcinogenesis, vol. 1, no. 6, pp. 495-499, 1980.

[26] F. W. R. Chaplen, "Incidence and potential implications of the toxic metabolite methylglyoxal in cell culture: a review," Cytotechnology, vol. 26, no. 3, pp. 173-183, 1998.

[27] M. Helal, M. Al-Douh, S. Hamid, H. Osman, S. Salhimi, and A. M. S. Abdul Majid, "Diaminobenzene schiff base induces caspase 9-dependent apoptosis in U937 leukemia cells," Cancer, vol. 1, no. 10, Article ID WMC001081, 2010.

[28] A. Jarrahpour, D. Khalili, E. De Clercq, C. Salmi, and J. M. Brunel, "Synthesis, antibacterial, antifungal and antiviral activity evaluation of some new bis-Schiff bases of isatin and their derivatives," Molecules, vol. 12, no. 8, pp. 1720-1730, 2007.

[29] M. Barber and G. A. D. Haslewood, "The antibacterial activity of simple derivatives of 2-aminophenol," Biochemical Journal, vol. 39, no. 4, pp. 285-287, 1945. 
[30] S. Sarkar and M. Akhter Farooque, "Antimicrobial and cytotoxic activities of 2-aminobenzoic acid and 2-aminophenol and their coordination complexes with Magnesium (Mg-II)," Pakistan Journal of Biological Sciences, vol. 7, pp. 25-27, 2004. 

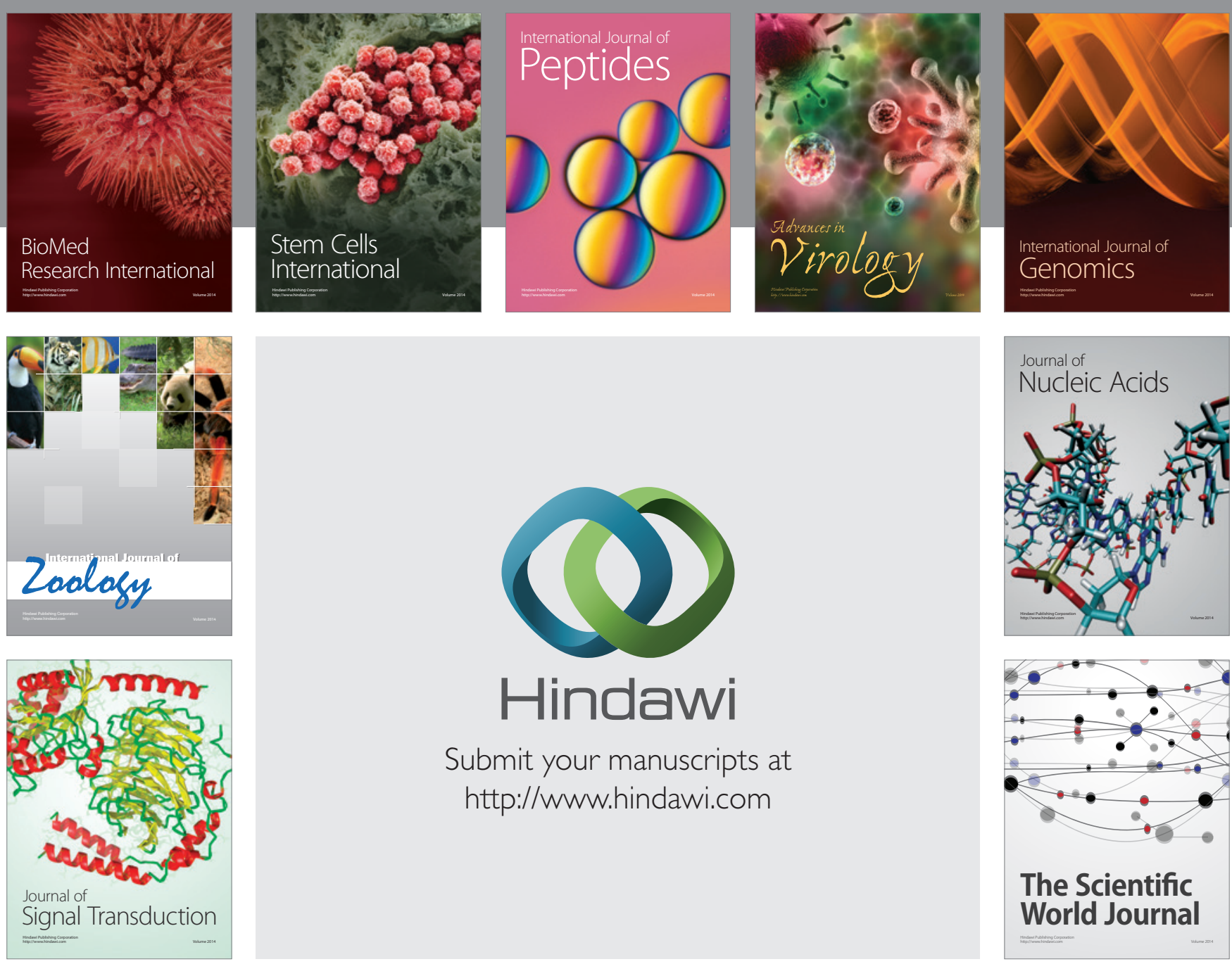

Submit your manuscripts at

http://www.hindawi.com
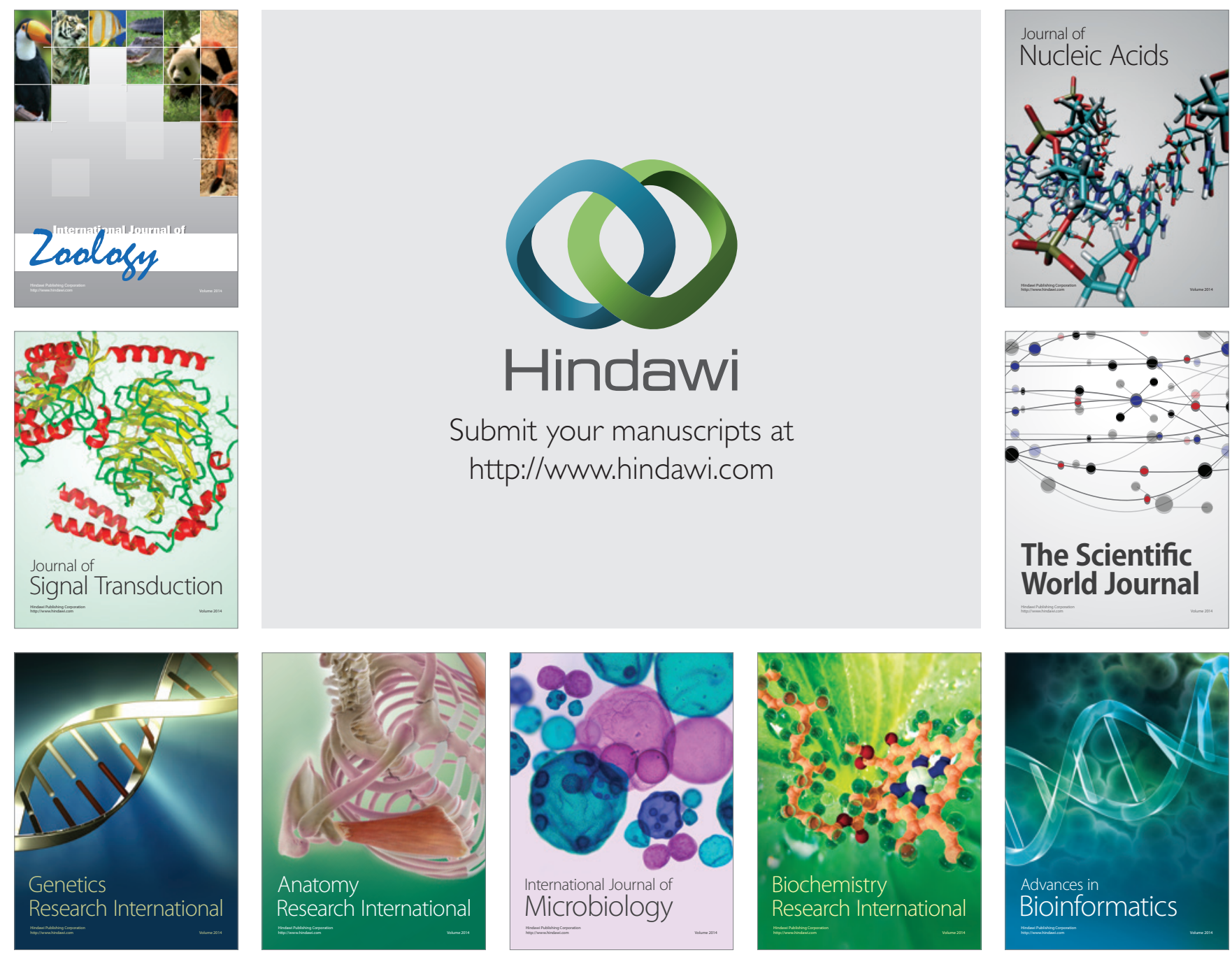

The Scientific World Journal
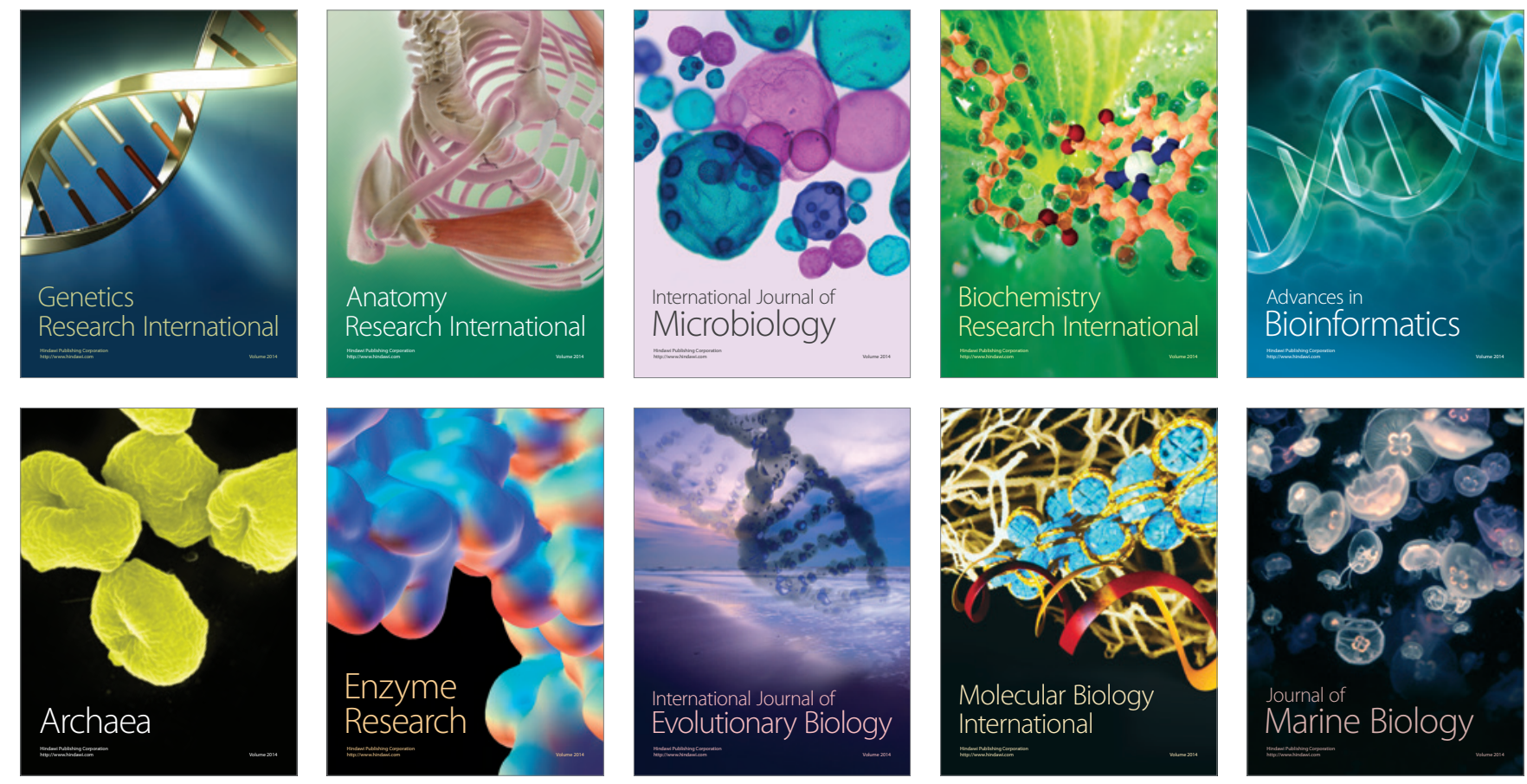